\title{
Multi-particle Correlations in Quaternionic Quantum Systems
}

\author{
S. P. Brumby* and G. C. Joshit \\ School of Physics, University of Melbourne, Research Centre for High Energy Physics, \\ Parkville, Victoria 3052, Australia. \\ R. Andersont \\ Dibner Institute for the History of Science and Technology, \\ 38 Memorial Drive, Massachusetts Institute of Technology, Cambridge, MA 02139, USA. \\ UM-P-94/54; RCHEP-94/15 \\ hep-th/9406040
}

\begin{abstract}
We investigate the outcomes of measurements on correlated, few-body quantum systems described by a quaternionic quantum mechanics that allows for regions of quaternionic curvature. We find that a multi-particle interferometry experiment using a correlated system of four nonrelativistic, spin-half particles has the potential to detect the presence of quaternionic curvature. Two-body systems, however, are shown to give predictions identical to those of standard quantum mechanics when relative angles are used in the construction of the operators corresponding to measurements of particle spin components.
\end{abstract}

PACS numbers: 03.65.Bz

Typeset using REVTEX 


\section{INTRODUCTION}

Quaternionic quantum mechanics (QQM) was raised as a possibility by Birkhoff and von Neumann [1] in 1936, and has been elaborated since then by a variety of authors using different theoretical approaches [2 9]. To date, however, the absence of clear experimental evidence has meant that researchers have constructed models with a concern to ensure that the quaternionic aspects are hidden in situations where standard, complex quantum mechanics (CQM) is successful. Fully quaternionic interactions are permitted, but the higher quaternionic components of the wavefunctions in these models exponentially decay in the absence of quaternionic-dissipative potentials [7]. Further, it is customary to work in what Finkelstein et al. [2] have classified as the Q-flat limit of the theory, thereby neglecting much of the geometrical content of the full theory.

Our contention is that within the general theory of QQM (General QQM) [2], which is the simplest example of a truly general implementation of QQM along the lines of Birkhoff's and von Neumann's work, the quaternionic nature of the states can manifest itself in collective, nonlocal effects.

In particular, there emerges a possibility of testing the prediction of General QQM that the $i$ of CQM becomes a field of pure imaginary unit quaternions on spacetime, by the simultaneous measurement at remote points of the intrinsic spin of particles in entangled states. In order to relate the complex algebras at each point, Ref. [2] uses concepts from differential geometry; gauge connections, covariant derivatives, and curvature have their quaternionic analogues in Q-connections, $\mathrm{Q}-$ covariant derivatives, and $\mathrm{Q}-$-curvature.

To treat many-body systems in their nonmutually interacting state (i.e., to construct Fock spaces of particles), we use the tensor product of quaternionic Hilbert modules developed by Horwitz and Razon [9]. This permits the definition of a scalar product of quaternionic multi-particle wavefunctions, by abandoning linearity in each factor of the tensor product in favour of a quotient-group structure, (we present a summary in the appendix).

In the next section, we examine the predictions of General QQM for the the archetypical 
experimental test (Bohm [10]) of the Einstein-Podolsky-Rosen programme (EPR) [11]. This was also the situation Bell [12] used in his famous demonstration that CQM could not be completed in accord with the EPR paper's assumptions.

This case raises a number of foundational questions about how we encode physical information in the specification of mathematical operators corresponding to particular measurements.

We then consider a similar experiment on a correlated four-electron state proposed by Greenberger et al. [13]. It is in this situation that we expect manifestations of quaternionic behaviour.

Given the enormous importance of the results engendered by Bell's work for exploring the foundations of CQM, we view the results of our paper as a preliminary contribution to locating their significance for exploring the foundations of QQM.

\section{TWO BODY CORRELATIONS}

Bohm's gedänkenexperiment consists of simultaneous spin component measurements on a system of two spin-half particles prepared in an entangled state

$$
|\Psi\rangle=\frac{1}{\sqrt{2}}\left(|+\rangle_{1}|-\rangle_{2}-|-\rangle_{1}|+\rangle_{2}\right)
$$

The preparation procedure, which is visualized as the decay out of a two-body S-state, results in a state which is rotationally invariant. That is, the complete entangled state is invariant under the group of rotations $\{g \otimes g \mid g \in \mathrm{SU}(2)\}$, where $\mathrm{SU}(2)$ is the covering group of $\mathrm{O}_{3}$. This is equivalent to stating that the measurement of internal spins of both particles along the same spatial axis, is independent of the orientation of that axis. (Note that this use of the quaternions, related to the spin-half nature of fundamental fermions, is located wholly within the domain of CQM). It is this invariance which essentially means we cannot ascribe any physical reality to definite spin components prior to their measurement.

The entangled character of the state is also invariant under this group, which implies 
that under linear, continuous evolution in free space, the state remains entangled and does not collapse into a trivial product of wavefunctions at distinct points.

In addition, this invariance means that when we measure spin components along different spatial directions, it is only the relative angular displacements of the analysing directions that are physically significant.

In CQM, this state can be multiplied by an additional arbitrary phase-factor which does not change the physical system (i.e. we actually deal with a equivalence class of states, of which the above is merely representative, the class relation being multiplication by a phasefactor). This enables us to choose the initial state Eq. (11) to be real, and in particular, to choose each factor to be real.

We now proceed to QQM, which is equivalent to a complete and distinct CQM at each point of spacetime, related to each other through the Q-connection. Hence it contains within it the property of local phase-invariance of states. In this case, the choice of a real initial state corresponds to nulling the phase-factors associated with each single particle wavefunction separately.

The $\mathrm{O}_{3}$ invariance of the quaternionic system, in the sense of spin measurements along the same spatial axis being independent of the orientation of that axis, is assured by the local equivalence between CQM and QQM. Hence the rotational invariance of the state still holds within QQM with the theory therefore still implying that individual particle spins fail to have any physical reality prior to their experimental resolution.

The Hermitian operator corresponding to the measurement, at position $\mathbf{x}$, of internal spin of a single particle, along a spatial unit vector

$$
\mathbf{n}=(\sin \theta \cos \phi, \sin \theta \sin \phi, \cos \theta),
$$

where the angles are defined with respect to some laboratory frame of reference, is

$$
\mathbf{n} \cdot \boldsymbol{\sigma}=\left(\begin{array}{cc}
\cos \theta & \sin \theta e^{-\phi \eta} \\
\sin \theta e^{\phi \eta} & -\cos \theta
\end{array}\right) .
$$


Here, $\eta$ is the quaternionic generalisation of the imaginary unit of CQM which, expanded with respect to a fixed basis of imaginary units, is

$$
\eta=\eta(\mathbf{x})=\sum_{r=1}^{3} h_{r}(\mathbf{x}) i_{r},
$$

where $\forall \mathbf{x}, \mathbf{h}(\mathbf{x}) \in \mathbf{R}^{3},\|\mathbf{h}\|=1$. That is, QQM requires the outcomes of measurements to depend on where they are carried out (phenomenon of Q-curvature).

The expectation value of the product of a component of each particles' spin,

$$
E_{\Psi}\left(\mathbf{n}, \mathbf{n}^{\prime}\right) \equiv \frac{1}{2}\left(P_{++}+P_{--}-P_{+-}-P_{-+}\right),
$$

which is equivalent to calculating

$$
E_{\Psi}\left(\mathbf{n}, \mathbf{n}^{\prime}\right)=\left\langle\Psi\left|\mathbf{n} \cdot \boldsymbol{\sigma}_{(1)} \otimes \mathbf{n}^{\prime} \cdot \boldsymbol{\sigma}_{(2)}\right| \Psi\right\rangle,
$$

where

$$
P_{+-}=\left\langle+\left|\left\langle-\left|\mathbf{n} \cdot \boldsymbol{\sigma}_{(1)} \otimes \mathbf{n}^{\prime} \cdot \boldsymbol{\sigma}_{(2)}\right|-\right\rangle\right|+\right\rangle .
$$

Note that the subscripts of $P$ refer to the first terms of each tensor product of states.

With the detectors at $\mathbf{x}_{1}$ and $\mathbf{x}_{2}$ respectively, without loss of generality we set $\eta\left(\mathbf{x}_{1}\right)=i_{1}$ and drop the position label from $\eta\left(\mathbf{x}_{2}\right)$.

$$
\begin{aligned}
P_{+-}= & \sum_{(\rho) \in \mathcal{I}^{2}}\left[\langle+|\langle-|\left(\sin \theta e^{\phi i_{1}}|+\rangle\right)_{\rho_{1}}\left(\sin \theta^{\prime} e^{-\phi^{\prime} \eta}|-\rangle\right)_{\rho_{2}}\right] \\
& \times\left(1 \otimes 1 \otimes 1 \cdot 1_{D(\mathbf{H})}, i_{\rho_{1}} \otimes i_{\rho_{2}} \otimes 1 \cdot 1_{D(\mathbf{H})}\right)_{\mathbf{H}} .
\end{aligned}
$$

For the reasons given previously, we consider the basis spin states $| \pm\rangle$ to be real. Then we can identify them with their formally real $0^{\text {th }}$ components (in the sense of [9])

$$
(| \pm\rangle)_{\sigma}=\frac{1}{4} \sum_{\varpi \in \mathcal{I}} i_{\varpi}\left(| \pm\rangle i_{\sigma}^{*}\right) i_{\varpi}^{*}=| \pm\rangle \delta_{\sigma 0} .
$$

Hence 


$$
\begin{aligned}
P_{+-}=\sin \theta \sin \theta^{\prime} & \sum_{\rho \in \mathcal{I}}\left(e^{-\phi^{\prime} \eta}\right)_{\rho} \\
\times & {\left[\cos \phi\left(1_{D(\mathbf{H})}, 1 \otimes i_{\rho} \otimes 1 \cdot 1_{D(\mathbf{H})}\right)_{\mathbf{H}}\right.} \\
& \left.+\sin \phi\left(1_{D(\mathbf{H})}, i_{1} \otimes i_{\rho} \otimes 1 \cdot 1_{D(\mathbf{H})}\right)_{\mathbf{H}}\right] .
\end{aligned}
$$

Applying the recursive definition to the inner product of tensor producted quaternionic algebras (given in the appendix), we have

$$
\begin{gathered}
\left(1_{D(\mathbf{H})}, 1 \otimes i_{\rho} \otimes 1 \cdot 1_{D(\mathbf{H})}\right)_{\mathbf{H}}=\frac{1}{3} i_{\rho}+\frac{2}{3} \delta_{\rho 0}, \\
\left(1_{D(\mathbf{H})}, i_{1} \otimes i_{\rho} \otimes 1 \cdot 1_{D(\mathbf{H})}\right)_{\mathbf{H}}=\frac{1}{3} \delta_{\rho 0} i_{1}-\frac{1}{3} \delta_{\rho 1} .
\end{gathered}
$$

Hence

$$
\begin{aligned}
P_{+-}=\sin \theta \sin \theta^{\prime}\left[\cos \phi \cos \phi^{\prime}+\frac{1}{3} \sin \phi \sin \phi^{\prime} h_{1}\right. \\
\left.+\frac{1}{3} \sin \phi \cos \phi^{\prime} i_{1}-\frac{1}{3} \cos \phi \sin \phi^{\prime} \eta\right] .
\end{aligned}
$$

Similarly,

$$
\begin{aligned}
P_{-+} & =\left\langle-\left|\left\langle+\left|\mathbf{n} \cdot \boldsymbol{\sigma}_{(1)} \otimes \mathbf{n}^{\prime} \cdot \boldsymbol{\sigma}_{(2)}\right|+\right\rangle\right|-\right\rangle, \\
& =\sin \theta \sin \theta^{\prime}\left(\left\langle-\left|\otimes\left\langle+\left|, e^{-\phi i_{1}}\right|+\right\rangle \otimes e^{\phi^{\prime} \eta}\right|-\right\rangle\right)_{\mathbf{H}} \\
& =P_{+-}\left(\phi \rightarrow-\phi ; \phi^{\prime} \rightarrow-\phi^{\prime}\right),
\end{aligned}
$$

so that in combination, we obtain the real result

$$
P_{+-}+P_{-+}=2 \sin \theta \sin \theta^{\prime}\left(\cos \phi \cos \phi^{\prime}+\frac{1}{3} \sin \phi \sin \phi^{\prime} h_{1}\right) .
$$

Now

$$
\begin{gathered}
P_{++}=\left\langle+\left|\left\langle-\left|\mathbf{n} \cdot \boldsymbol{\sigma}_{(1)} \otimes \mathbf{n}^{\prime} \cdot \boldsymbol{\sigma}_{(2)}\right|+\right\rangle\right|-\right\rangle, \\
P_{--}=P_{++}=-\cos \theta \cos \theta^{\prime} .
\end{gathered}
$$


Therefore we have the (real) expectation value

$$
\begin{aligned}
E_{\Psi}\left(\mathbf{n}, \mathbf{n}^{\prime}\right)= & -\cos \theta \cos \theta^{\prime} \\
& -\sin \theta \sin \theta^{\prime}\left(\cos \phi \cos \phi^{\prime}+\frac{1}{3} h_{1} \sin \phi \sin \phi^{\prime}\right) .
\end{aligned}
$$

The final term in this sum represents the manifestation of QQM in this nonrelativistic, cor-

related quantum system. It is a specialisation of the Euclidean inner product $\mathbf{h}\left(\mathbf{x}_{1}\right) \cdot \mathbf{h}\left(\mathbf{x}_{2}\right)$, arising from the natural inner product structure imposed upon the quaternionic algebra.

As stated before, rotational invariance of the initial entangled state Eq. (11) requires the use of relative angles for the phases $\phi, \phi^{\prime}$, (i.e., $\phi \rightarrow \phi_{\text {rel }}=\phi-\phi^{\prime}, \phi^{\prime}=0$ ), Hence, we retrieve the CQM result

$$
E_{\Psi}^{0}\left(\mathbf{n}, \mathbf{n}^{\prime}\right)=-\cos \theta \cos \theta^{\prime}-\sin \theta \sin \theta^{\prime} \cos \phi_{\text {rel }}
$$

That is, by defining a polar axis, $\phi=\phi^{\prime}=0$, we have

$$
E_{\Psi}\left(\mathbf{n}, \mathbf{n}^{\prime}\right)=-\cos \theta_{\text {rel }}=-\mathbf{n} \cdot \mathbf{n}^{\prime}
$$

Alternatively, restricting the $\mathbf{n}$ 's to a plane, $\theta=\theta^{\prime}=\pi / 2$, implies

$$
E_{\Psi}\left(\mathbf{n}, \mathbf{n}^{\prime}\right)=-\cos \phi_{\text {rel }}=-\mathbf{n} \cdot \mathbf{n}^{\prime}
$$

The fact that general QQM hides itself at this level of two-body systems is an interesting result, especially as it tends to imply that QQM might not manifest itself in models neglecting $\geq 3$-body interactions. This would suggest that the present lack of experimental evidence for QQM may be due to the subtlety of the theory.

\section{FOUR BODY CORRELATIONS}

We now consider a 4-particle correlated system described by Greenberger et al. [13].

$$
\left|\Psi_{\mathrm{GHSZ}}\right\rangle=\frac{1}{\sqrt{2}}\left(|+\rangle_{1}|+\rangle_{2}|-\rangle_{3}|-\rangle_{4}-|-\rangle_{1}|-\rangle_{2}|+\rangle_{3}|+\rangle_{4}\right)
$$


We carry out simultaneous (or at least, space-like separated) measurements of a component of spin of each particle, and consider the product of these values. The quantum mechanical expectation value is thus

$$
\begin{gathered}
E_{\mathrm{GHSZ}}\left(\left\{\mathbf{n}_{(j)}\right\}\right)=\left\langle\Psi_{\mathrm{GHSZ}}\left|\bigotimes_{j=1}^{4} \mathbf{n}_{(j)} \cdot \boldsymbol{\sigma}_{(j)}\right| \Psi_{\mathrm{GHSZ}}\right\rangle \\
P_{+-}=\left\langle+\left|\left\langle+\left|\left\langle-\left|\left\langle-\left|\bigotimes_{j=1}^{4} \mathbf{n}_{(j)} \cdot \boldsymbol{\sigma}_{(j)}\right|-\right\rangle\right|-\right\rangle\right|+\right\rangle\right|+\right\rangle \\
P_{+-}=\left(\prod_{j=1}^{4} \sin \theta_{j}\right) \sum_{(\rho) \in \mathcal{I}^{4}}\left(e^{\phi_{1} \eta\left(\mathbf{x}_{1}\right)}\right)^{\rho_{1}}\left(e^{\phi_{2} \eta\left(\mathbf{x}_{2}\right)}\right)^{\rho_{2}}\left(e^{-\phi_{3} \eta\left(\mathbf{x}_{3}\right)}\right)^{\rho_{3}}\left(e^{-\phi_{4} \eta\left(\mathbf{x}_{4}\right)}\right)^{\rho_{4}}\left(1_{D(\mathbf{H})}, \mathrm{i}_{(\rho)} \otimes 1 \cdot 1_{D(\mathbf{H})}\right) \mathbf{H}
\end{gathered}
$$

where $\left\{\theta_{j}, \phi_{j}\right\}$ are the polar and azimuthal angles specifying the direction $\mathbf{n}_{j}$ along which a measurement is made on the $j^{\text {th }}$ particle.

We now simplify this expression by considering the special configuration $\forall j, \theta_{j}=0$ and without loss of generality take $\eta_{3}=i_{1}$. Further, we use relative angles: $\forall j, \phi_{j} \rightarrow \phi_{j}-\phi_{4}$.

Hence,

$$
\begin{aligned}
P_{+-}=\sum_{(\rho) \in \mathcal{I}^{2}}\left(e^{\phi_{1} \eta\left(\mathbf{x}_{1}\right)}\right)^{\rho_{1}}\left(e^{\phi_{2} \eta\left(\mathbf{x}_{2}\right)}\right)^{\rho_{2}}[ & \cos \phi_{3}\left(1_{D(\mathbf{H})}, \mathrm{i}_{(\rho)} \otimes 1 \otimes 1 \otimes 1 \cdot 1_{D(\mathbf{H})}\right)_{\mathbf{H}} \\
& \left.-\sin \phi_{3}\left(1_{D(\mathbf{H})}, \mathrm{i}_{(\rho)} \otimes i_{1} \otimes 1 \otimes 1 \cdot 1_{D(\mathbf{H})}\right) \mathbf{H}\right] .
\end{aligned}
$$

Using the recursive definition for the inner-product of quaternion algebras, we obtain from Eqs. (20)-(24),

$$
\begin{aligned}
E_{\mathrm{GHSZ}}\left(\left\{\mathbf{n}_{(j)}\right\}\right)= & -\frac{1}{2}\left(P_{+-}+P_{-+}\right) \\
= & -\cos \phi_{1} \cos \phi_{2} \cos \phi_{3}-\frac{1}{3} h_{1}\left(\mathbf{x}_{1}\right) \sin \phi_{1} \cos \phi_{2} \sin \phi_{3}-\frac{1}{3} h_{1}\left(\mathbf{x}_{2}\right) \cos \phi_{1} \sin \phi_{2} \sin \phi_{3} \\
& +\frac{1}{3} \mathbf{h}\left(\mathbf{x}_{1}\right) \cdot \mathbf{h}\left(\mathbf{x}_{2}\right) \sin \phi_{1} \sin \phi_{2} \cos \phi_{3} .
\end{aligned}
$$

Hence, we find that QQM effects are manifest in experiments on this 4-body correlated system. Moreover, we expect QQM to give different predictions to CQM for all $N \geq 2$ body system. (On this point, we mention that Horwitz and Razon [9] have suggested that as 
$N \rightarrow \infty$, with their definition of a multi-particle inner-product, we recover the quantizedfield commutator relations of complex quantum field theory).

The CQM expectation value is

$$
E_{\mathrm{GHSZ}}^{0}\left(\left\{\mathbf{n}_{(j)}\right\}\right)=-\cos \left(\phi_{1}+\phi_{2}-\phi_{3}\right)
$$

Comparing this with Eq. (25), we see that the CQM limit is reached by nulling the Qcurvature and removing the numerical factors that arise from the recursive definition of the scalar product.

$$
\begin{gathered}
\forall \mathbf{x}, \mathbf{x}^{\prime} \quad \frac{1}{3} \mathbf{h}(\mathbf{x}) \cdot \mathbf{h}\left(\mathbf{x}^{\prime}\right) \rightarrow 1, \\
\text { or, } \forall \mathbf{x}, h_{1}(\mathbf{x})=\sqrt{3}, h_{2}(\mathbf{x})=h_{3}(\mathbf{x})=0,
\end{gathered}
$$

where in the latter we have gone back to Eq. (24) and taken $h_{1}\left(\mathbf{x}_{3}\right)=1 \rightarrow \sqrt{3}$. This "rescaling" of the $\eta$ field might possibly be avoided by using a different definition for the inner product, but the occurrence of $\mathbf{h}(\mathbf{x}) \cdot \mathbf{h}\left(\mathbf{x}^{\prime}\right)$ coefficients to the relative-orientation dependent terms in the expectation value might still be expected (the same terms arise in the simplest approaches to this calculation [14]).

Note that if instead we considered an experiment which made use of plane polarised photons, in the manner described by Ref. [13], then because photons possess a single quantum of angular momentum, the operator corresponding to a polarisation filter has real components 15] and QQM has no opportunity to manifest itself. This situation is changed by the use of circularly polarised photons, in which case the quantum mechanical prescription for calculating the expectation values necessarily uses complex numbers, giving the quaternionic entangled state a probability distribution sensitive to a changing $\eta$-field (hence, not predicted by CQM).

\section{CONCLUSION}

We see that it is an artifice of particle number which hides QQM in two-body situations. For more complicated systems, General QQM gives expectation values that differ formally 
from those of CQM. The importance of particle number in this situation is analogous to that pertaining to correlations in CQM. As Greenberger et al. have shown, Bell type inequalities which permit local realistic hidden variable theories to agree with quantum mechanical predictions in certain regions of parameter space (though not in all regions) only occur for two-particle entangled states. For more complicated systems, however, CQM predicts correlations for entangled systems which are unable to be described by local hidden variable theories.

Our result in Eq. (25) indicates that experimental tests of 4-particle correlations have either the potential to reveal quaternionic components or to set limits on their values. Our prediction of quaternionic terms in multiparticle correlation experiments provides a further motivation to the reasons given by Greenberger et al. to undertake such experiments.

If such an experimental test of QQM were carried out, and no quaternionic correction terms were discovered, then in some clear sense we would have evidence against what we would maintain is the most natural interpretation and implementation of the suggestion of Birkhoff and von Neumann.

QQM may still be the "real" theory of quantum mechanics, but its contribution to phenomena at human scales, and in our vicinity of spacetime, may be slight. In this case until there occurs a situation where QQM provides an explanation analogous in significance to General Relativity's explanation of the anomalous precession of Mercury, the theory will remain tentative. (This raises an obvious question about what could act as a strong source of Q-curvature, to which no answer has yet been proposed in the literature.)

A more modest perspective would be to sidestep for the moment questions as to whether or not QQM is the "real theory" of quantum phenomena, and simply to view QQM in a phenomenological sense as a theory whose extra parameters affords a way of better capturing experiment results that have defied explanation by other means. The results of the type we present in Sec. III indicate the manner in which this parametrization can occur. Naturally any success on this score invites a deeper consideration on the viability of QQM.

Depending on the outcomes of these possibilities, QQM may tell us something crucial 
about the necessity of both q-numbers and c-numbers in the correct description of physical phenomena as we know them. QQM exists as a potential alternative theory because of the correspondence we set up between experimentally observable quantities and Hermitian operators (which necessarily possess real eigenvalues). If experiments fail to support General QQM, then we have found out something about the physical standing of non-Hermitian operators and unobservable properties of a system.

We await experimental clarification of these concerns.

\section{ACKNOWLEDGMENTS}

One of the authors, S. P. B., acknowledges the support of an Australian Postgraduate Research Award. G. C. J. was supported by the Australian Research Council and the University of Melbourne. R. A. acknowledges the support provided by the Dibner Institute, and is grateful for the hospitality provided by the Institute.

\section{APPENDIX: TENSOR PRODUCT OF QUATERNION ALGEBRAS}

$\mathbf{R}$ the real algebra

$\mathbf{H}$ the quaternion algebra

$1, i_{1}, i_{2}, i_{3} \quad$ a basis for $\mathbf{H}$, where $\forall r, s \in\{1,2,3\}$,

$$
i_{r} i_{s}=-\delta_{r s}+\sum_{t=1}^{3} \epsilon_{r s t} i_{t}
$$

$\operatorname{Im} \mathbf{H}$ the set $\{\eta\}$ of imaginary elements of $\mathbf{H}$,

$$
\eta=\sum_{r=1}^{3} h_{r} i_{r} \text { where } \mathbf{h} \in \mathbf{R}^{3},\|\mathbf{h}\|=1
$$

$\mathcal{H}_{\mathbf{H}}$ a Hilbert $\mathbf{H}$-module

$\mathbf{C}_{\eta} \quad$ the complex algebra generated by 1 and $\eta$

$D \quad$ the real linear span $\mathbf{H} \otimes \cdots \otimes \mathbf{H}$

$\mathcal{I}$ the set $\{0,1,2,3\}$

$(\rho) \quad$ an element $\left(\rho_{1}, \ldots, \rho_{N}\right) \in \mathcal{I}^{N}$ 
$\mathrm{i}_{(\rho)} \quad$ the tensor product $i_{\rho_{1}} \otimes \cdots \otimes i_{\rho_{N}}$, where $i_{0}=1$

[q] the product $q \otimes \cdots \otimes q \in D$

$[q]_{j} \quad$ the product $1 \otimes \cdots \otimes q \otimes \cdots \otimes 1 \in D$, with the $q$ in the $j$ th place

$(q)_{0} \quad$ the real component of $q \in \mathbf{H}$

$(q)_{\eta} \quad$ the $\mathbf{C}_{\eta}$ component of $q$

$(\mathbf{q})_{0} \quad\left(q_{1}\right)_{0} \cdots\left(q_{N}\right)_{0}$ for $\mathbf{q}=q_{1} \otimes \cdots \otimes q_{N} \in D$

$(\mathbf{q})_{\eta} \quad\left(q_{1}\right)_{\eta} \ldots\left(q_{N}\right)_{\eta}$

$A_{\eta} \quad$ a left ideal of $D$ generated by the set

$$
\left\{[\eta]_{1}-[\eta]_{j} \mid 2 \leq j \leq N\right\}
$$

$D(\eta) \quad$ the quotient $D / A_{\eta}$

$1_{D} \quad 1 \otimes \cdots \otimes 1 \in D$

$1_{D(\eta)} \quad 1_{D}+A_{\eta}$

$A_{\mathbf{H}} \bigcap_{\eta \in \operatorname{Im} \mathbf{H}} A_{\eta}$

$D(\mathbf{H}) \quad$ the quotient $D / A_{\mathbf{H}}$

$1_{D(\mathbf{H})} \quad 1_{D}+A_{\mathbf{H}}$

We define pairwise multiplication in the product algebra,

$$
\begin{aligned}
\mathrm{qr} & =\left(q_{1} \otimes \cdots \otimes q_{N}\right)\left(r_{1} \otimes \cdots \otimes r_{N}\right) \\
& =\left(q_{1} r_{1} \otimes \cdots \otimes q_{N} r_{N}\right) .
\end{aligned}
$$

$(\mathrm{q})_{\mathcal{Q}}$ is defined inductively for $\mathrm{q} \in D$ by

$$
\left(q_{1}\right)_{\mathcal{Q}}=\left(q_{1}\right)_{0}=\operatorname{Re} q_{1},
$$

and for $N>1$, 


$$
\begin{gathered}
(\mathbf{q})_{\mathcal{Q}}=\frac{1}{N+1}\left[\left(q_{1} q_{N} \otimes \cdots \otimes q_{N-1}\right)_{\mathcal{Q}}\right. \\
+\cdots+\left(q_{1} \otimes \cdots \otimes q_{N-1} q_{N}\right)_{\mathcal{Q}} \\
\left.+2\left(q_{N}\right)_{0}\left(q_{1} \otimes \cdots \otimes q_{N-1}\right)_{\mathcal{Q}}\right] . \\
\left(\mathbf{q}_{1} \cdot 1_{D(\mathbf{H})}, \mathbf{q}_{2} \cdot 1_{D(\mathbf{H})}\right)_{\mathbf{H}}=\sum_{j=0}^{3}\left(\mathbf{q}_{1}^{*}\left[i_{j}^{*}\right]_{N} \mathbf{q}_{2}\right)_{\mathcal{Q}} i_{j} .
\end{gathered}
$$

For each $\psi \in \mathcal{H}_{\mathbf{H}}$, the formally real components of $\psi$ are defined at each point $\mathbf{x}$ by

$$
\begin{aligned}
(\psi)_{\mathbf{H}} & =\sum_{\sigma \in \mathcal{I}}\left(\psi \otimes i_{\sigma}^{*}(\mathbf{x})\right)_{\mathcal{Q}} i_{\sigma}(\mathbf{x}) \\
& =\sum_{\sigma \in \mathcal{I}} \psi_{\sigma}^{\mathbf{x}} i_{\sigma}(\mathbf{x})
\end{aligned}
$$

We now choose to decompose each tetrad $i_{\sigma}(\mathbf{x})$ as a rotation from a standard basis $i_{\rho}$ (the rotation being an element of the group $\left.\mathrm{I}_{1} \oplus \mathrm{O}_{3}\right)$.

$$
(\psi)_{\mathbf{H}}=\sum_{\rho \in \mathcal{I}}\left(\sum_{\sigma \in \mathcal{I}} \psi_{\sigma}^{\mathbf{x}} \mathcal{O}_{\sigma \rho}(\mathbf{x})\right) i_{\rho}=\sum_{\rho \in \mathcal{I}} \psi_{\rho} i_{\rho}
$$

We can now form the quaternion tensor product of $N$ single particle systems, which is itself a left module over the quaternionic algebra:

$$
\Psi q=\psi^{1} \otimes \cdots \otimes \psi^{N} \otimes q \in \mathcal{H}_{\mathbf{H}}^{N} \otimes \mathbf{H}
$$

As usual, for an interacting system, the states of the $N$-body interacting quantum system can be decomposed in the basis formed by the $N$-tensor product of a basis $\mathbf{B}\left(\mathcal{H}_{\mathbf{H}}\right)$ for $\mathcal{H}_{\mathbf{H}}$.

Using the decomposition into formally real components of each single particle wavefunction,

$$
\begin{aligned}
\Psi & =\sum_{(\rho) \in \mathcal{I}^{N}}\left(\psi_{\rho_{1}}^{1} i_{\rho_{1}}\right) \otimes \cdots \otimes\left(\psi_{\rho_{N}}^{N} i_{\rho_{N}}\right) \otimes 1 \cdot 1_{D(\mathbf{H})} \\
& =\sum_{(\rho) \in \mathcal{I}^{N}}\left(\psi_{\rho_{1}}^{1} \otimes \cdots \otimes \psi_{\rho_{N}}^{N}\right) \otimes\left(\mathrm{i}_{(\rho)} \otimes 1 \cdot 1_{D(\mathbf{H})}\right),
\end{aligned}
$$

where we have taken the formally real components and formed them into a correspondingly ordered $N$-tuple of functions. 
Thus, $\Psi \in\left[\mathcal{L}^{2}\left(\mathbf{R}^{d}\right)\right]^{N} \otimes D^{(N+1)}(\mathbf{H})$, where $\mathcal{L}^{2}\left(\mathbf{R}^{d}\right)$ is the set of real, Lebesgue squareintegrable functions on $d$-dimensional Euclidean space (for particles in a box, these functions are of finite support).

We can now define a scalar product of $N$-body wavefunctions:

$$
\begin{array}{r}
\langle\Psi, \Phi\rangle_{\mathbf{H}}=\sum_{(\rho),(\sigma) \in \mathcal{I}^{N}}\left\langle\psi_{\rho_{1}}^{1} \otimes \cdots \otimes \psi_{\rho_{N}}^{N} \mid \phi_{\sigma_{1}}^{1} \otimes \cdots \otimes \phi_{\sigma_{N}}^{N}\right\rangle \\
\times\left(\mathrm{i}_{(\rho)} \otimes 1 \cdot 1_{D(\mathbf{H})}, \mathrm{i}_{(\sigma)} \otimes 1 \cdot 1_{D(\mathbf{H})}\right)_{\mathbf{H}} .
\end{array}
$$




\section{REFERENCES}

* e-mail: spb@tauon.ph.unimelb.EDU.AU.

† e-mail: joshi@tauon.ph.unimelb.EDU.AU.

$\ddagger$ e-mail: randerso@MIT.EDU. Permanent address: Department of Philosophy, Boston College, Chestnut Hill, MA 02167.

[1] G. Birkhoff and J. von Neumann, Ann. Math. 37, 823 (1936).

[2] D. Finkelstein, J. M. Jauch, S. Schiminovich and D. Speiser, J. Math. Phys. 3, 207 (1962); 4, 788 (1963).

[3] A. Peres, Phys. Rev. Lett. 42, 683 (1979).

[4] S. L. Adler, Phys. Rev. D 21, 2903 (1980).

[5] L. P. Horwitz and L. C. Biedenharn, Ann. Phys. 157, 432 (1984).

[6] C. G. Nash and G. C. Joshi, Int. J. Theor. Phys. 31, 965 (1992); 27, 409 (1988); J. Math. Phys. 28, 2883 (1987); 28, 2886 (1987).

[7] S. L. Adler, Phys. Rev. Lett. 57, 167 (1986); Commun. Math. Phys. 104, 611 (1986); Phys. Rev. D 37, 3654 (1988); L. P. Horwitz, J. Math. Phys. 34, 3405 (1993).

[8] A. Davies and B. H. J. McKellar, Phys. Rev. A 40, 4209 (1989); A. Davies, Phys. Rev. D 41, 2628 (1990).

[9] A. Razon and L. P. Horwitz, Acta Appl. Math. 24, 141 (1991); 24, 179 (1991).

[10] D. Bohm, Quantum Theory (Prentice-Hall, New York, 1951), Chap. 22, Sec. 15-19.

[11] A. Einstein, B. Podolsky and N. Rosen, Phys. Rev. 47, 777 (1935).

[12] J. S. Bell, Physics 1, 195 (1964).

[13] D. M. Greenberger, M. Horne, and A. Zeilinger, in Bell's Theorem, Quantum Theory, 
and Conceptions of the Universe, edited by M. Kafatos (Kluwer Academic, Dordrecht, The Netherlands, 1989), p. 73; D. M. Greenberger, M. A. Horne, A. Shimony and A. Zeilinger, Am. J. Phys. 58, 1131 (1990).

[14] S. P. Brumby, B.Sc.(Hons) thesis, Melbourne University, 1993.

[15] J. F. Clauser and A. Shimony, Rep. Prog. Phys. 41, 1881 (1978). 